\title{
Percepciones de los docentes y directivos sobre los procesos de transformación de los planes de mejoramiento institucional
}

\author{
Perceptions of teachers and school chiefs, about the \\ transformation processes of institutional improvement plans
}

\author{
Jorge Eliecer Torres Argumedo \\ Universidad de Córdoba, Colombia \\ jetarr@hotmail.com
}

\author{
Rafael Díaz Pérez \\ Universidad de Córdoba, Colombia \\ rafadipe10@hotmail.com
}

Palabras claves:

Percepciones, Plan de Mejoramiento Institucional, Calidad Educativa, Autoevaluación Institucional, PEI.

\section{Resumen}

Este artículo devela las percepciones docentes y directivos, sobre los procesos de transformación de los Planes de Mejoramiento Institucional (PMI). Parte del análisis reflexivo y colectivo de los PMI de las instituciones educativas Policarpa Salavarrieta y Victoria Manzur de Montería, para la generación de acciones conducentes al fortalecimiento de los procesos institucionales y pedagógicos. Se empleó un método de investigación cualitativa, con diseño metodológico Investigación-Acción, organizado en cuatro fases: aproximación que da origen a los propósitos investigativos, producto de la revisión y análisis de los PMI; reflexión proporcionó pautas para el diseño de plan de acción, producto del grupo de discusión y el fortalecimiento del marco teórico; aplicación como un acercamiento a la realidad de los PMI y su relevancia en las instituciones educativas, finalmente la evaluación, que proporciona el resultado obtenido. El estudio muestra variadas percepciones sobre los PMI, en relación a la participación de los docentes y los directivos, evidenciando un bajo nivel de actuación; pero, la dinámica implementada, permitió vislumbrar cambios en la adopción, elaboración y ejecución de los PMI, permitiendo ir dándole un nuevo sentido a las acciones de mejora en los establecimientos educativos objeto de estudio.

Recepción: Dic 19 de 2019 | Aceptación: Mar 05 de 2020 | Publicación: Dic 15 de 2020 


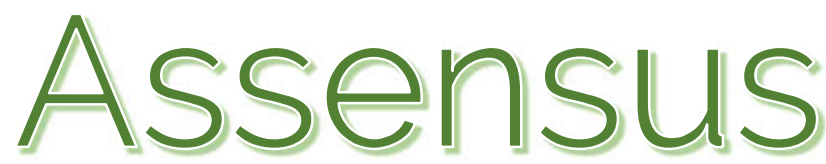

Revista de Investigación educativa y pedagógica

\begin{tabular}{ll}
\hline & Abstract \\
& This article shows the teaching and managerial perceptions of the \\
transformation processes of the Institutional Improvement Plans \\
(PMI). Part of the reflective and collective analysis of the PMls of \\
the educational institutions Policarpa Salavarrieta and Victoria \\
Manzur de Monteria, for the generation of actions leading to the \\
strengthening of the institutional and pedagogical processes. A \\
qualitative research method was used, with a Research-Action \\
methodological design, organized in four phases: an approach \\
that gives rise to research purposes, as a result of the review and \\
analysis of PMls; Reflection provided guidelines for the design of \\
an action plan, product of the discussion group and the \\
strengthening of the theoretical framework; application as an \\
approach to the reality of the PMl and its relevance in educational \\
institutions, finally the evaluation, which provides the result \\
obtained. The study shows varied perceptions about the PMl, in \\
relation to the participation of teachers and managers, showing a \\
low level of performance; but, the dynamics implemented, \\
Keywords:
\end{tabular}

Assessment, PEI.

Received: Dec 19, 2019 | Accepted: Mar 05, 2020 | Published: Dec 15, 2020 


\section{Introducción}

La educación colombiana en la primera década del siglo XXI se ha sustentado bajo el modelo de gestión de calidad, plasmado en la política de revolución educativa con el propósito de "apoyar a las entidades territoriales y a los establecimientos educativos en sus procesos de mejoramiento institucional orientados a lograr que los educandos obtengan mejores resultados en competencias básicas, laborales y valores ciudadanos" (Altablero, 2007, pág. 1). Lo anterior, implica impulsar procesos de descentralización que transfieren responsabilidades administrativas y financieras a las instancias educativas locales, acompañados de sistemas de información para la gestión; otorgar autonomía a las instituciones educativas bajo lineamientos definidos por el Ministerio de Educación Nacional que, como ente rector del sistema, debe garantizar su cohesión; y establecer un sistema de evaluación para medir los resultados de la calidad educativa e impulsar su mejoramiento, y diseñar mecanismos de rendición de cuentas y responsabilidad por los resultados (Castañeda, 2009).

A partir del año 2010 con proyección a 2020, Colombia, en materia educativa asumió un rol formativo diferente tomando como prioridad el mejoramiento relacionado con la prosperidad económica y social. De ahí que en el plan de desarrollo se incluyan mayores recursos para fortalecer la educación en los niveles de preescolar, básica y media (Fundación Empresarios por la Educación, 2018). Esto demuestra que en los últimos ocho (8) años se ha fortalecido el ofrecimiento de una educación incluyente y de calidad, priorizando la educación inicial, en la cual se garantiza mayor tiempo de los niños y jóvenes en los establecimientos educativos. Igualmente, se incluyen mecanismos de calidad, formando y mejorando la profesión docente. Todo esto, como reto de proyectar una mejor calidad educativa.

Con relación a las políticas de calidad, las instituciones educativas oficiales y privadas de Colombia, están en el deber, según su autonomía, de poner en marcha un Plan de Mejoramiento Institucional que se proyecte a la mejora de la calidad educativa, mediante la dinámica de programaciones, acciones y el establecimiento de metas previamente planeadas, organizadas y sistematizadas, los cuales debe ser realizados por la comunidad educativa y liderado por el rector, acompañado por el Consejo Directivo y Académico, involucrando a los docentes, padres de familia y estudiantes (MEN, 2004).

De esta manera, el Ministerio de Educación Nacional de Colombia, propone una herramienta orientadora para que cada establecimiento educativo dinamice sus planes de mejoramiento, derivado de la autoevaluación institucional contemplada en la guía 34 que orienta e invita a recopilar, sistematizar, analizar y valorar la información sobre el desarrollo de las acciones y del resultado de los procesos del establecimiento (MEN, 2008). Dentro de los puntos que se involucra se encuentra la gestión directiva, la académica, la administrativa 
y financiera y la gestión de la comunidad; lo destacado de ello, es que cuando se hace la autoevaluación institucional, de allí surgen los planes de mejoramiento.

No obstante, a pesar de que hace más de 14 años se desarrolla el ejercicio de elaboración del Plan de Mejoramiento Institucional (PMI) en muchas instituciones, no se evidencia la mejora en los establecimientos educativos, en materia institucional y pedagógica. Lo anterior genera grandes inquietudes en la comunidad educativa, que se reflejan en la poca participación de los docentes en el diseño de los programas de mejoramiento institucional. También se muestran descontextualizados y carente de transformaciones en el ámbito escolar, debido al limitado compromiso docente, poco liderazgo y la falta de una actitud de transformación en los recintos escolares.

En este sentido, desde este artículo se analizan las percepciones de los docentes y directivos docentes acerca de los procesos de participación y transformación de dichos planes, así como su aporte al fortalecimiento de los procesos institucionales. Esto conlleva hacer algunas acciones como la de interpretar las percepciones y el nivel de participación de los actores educativo en los PMI, y el efecto de estos en la transformación de los establecimientos educativos; para ello, se diseñan e implementan acciones que orientan su resignificación y desarrollo en los procesos de seguimiento, con la firme intención de determinar los cambios en la práctica pedagógica derivada de la implementación de los PMI.

Hay que entender, que los planes de mejoramiento hacen parte de un proceso administrativo "nuevo" para las instituciones educativas que se encuentran en desarrollo, perfeccionamiento y crecimiento constante, por lo cual se requiere que los implicados en ello, asuman su rol proactivo en la transformación y evolución de dichos planes, a partir de construcciones epistemológicas que favorezcan la práctica educativa que se desarrolla en las aulas, la escuela y la comunidad, incluyendo en este proceso de desarrollo institucional, a todos los factores que puedan contribuir con estas transformaciones.

De ahí, que este documento interpreta, mediante procesos de autorreflexión y desde un nivel comprensivo, las percepciones de los docentes y directivos docentes sobre los procesos de transformación de los PMI en las Instituciones Educativas, para facilitar el diseño de estrategias pedagógicas que permitan el fortalecimiento y desarrollo proactivo del quehacer docente. Por lo tanto, el estudio va ligado a la comprensión que tienen los docentes sobre la importancia del plan de mejoramiento y cómo llega a fortalecer la labor de su rol en la práctica pedagógica, es decir, ¿cómo el docente percibe el plan de mejoramiento de su institución?, ¿cuál es su rol en el desarrollo de dicha actividad? y ¿cómo los hallazgos y el desarrollo del proceso le brindan la posibilidad de construir nuevas configuraciones epistemológicas que potencialicen su ejercicio pedagógico y su rol en la institución?. 


\section{Referentes teóricos}

Muchas investigaciones reconocen la importancia de la educación en términos de gestión, relacionados con los procesos de transformación y participación docente en el desarrollo institucional. Para ello, se hizo una revisión de antecedentes relacionada con los procesos de mejoramiento institucional; en este sentido, Avaca y De León (2012) nos relatan los obstáculos y desafíos que enfrenta el docente y los directivos docentes en la dinámica de implementación de los PMl; para ello, se proponen espacios de participación a los miembros de la comunidad escolar tanto en el diseño como en la implementación y evaluación de los planes de mejora.

Otro aspecto importante lo concibe Rosero (2012), quien sostiene que las percepciones y prácticas de gestión, señalan realidades sobre el liderazgo de las instituciones educativas, desde lo fundamental como son las concepciones, emociones e intereses y las acciones técnico operativas que definen el proceso de dirección institucional; la importancia de la investigación es su relación con la calidad de los procesos de mejoramiento institucional y educativo; el plan de mejoramiento que una institución determinada se plantea a corto plazo, dejando de lado los procesos complejos a mediano y largo plazo y enfatizando en la relación entre funciones y necesidades donde directivos y docentes son participes de los procesos que se planifiquen y ejecuten según necesidades y particularidades de las instituciones.

La propuesta de Ojeda \& Romero (2014), apunta al establecimiento sobre la incidencia del Plan de Mejoramiento Institucional en el fortalecimiento de la calidad educativa desde la construcción colectiva, que aborda la dinámica del sector educativo en el desarrollo de las comunidades lo cual facilitaría la adecuada interrelación docente - alumno - padre de familia, contribuyendo a la mejora, apropiación y aplicación de PMI hacia la construcción de una ruta consecuente de calidad en la que los docentes y directivos se interrelacionan en el proceso educativo institucional. Otro aspecto importante lo contempla el informe investigativo "PMI, pensados desde el liderazgo que implica la gestión educativa" llevado a cabo por Castañeda (2015), el cual expresa que los rectores como líderes y gestores sociales junto con los docentes, direccionan de manera conjunta diferentes propuestas, estrategias y acciones, que involucren de manera concreta a estudiantes y padres de familia en los procesos de mejoramiento, de esta manera su impacto no se limita al espacio escolar como tal, sino que trasciende a la cultura, a la sociedad en la cual se encuentra inmerso el establecimiento educativo.

Por lo anterior, se toma el aporte gerencial dado como referente, porque este constituye el camino para alcanzar el éxito, pero para poder alcanzarlo, es necesario partir del liderazgo ejercido por el directivo docente, que debe promover la calidad educativa y la autonomía escolar a través de una visión clara sobre el papel que cumplen los planes de mejoramiento institucional, la evaluación y la autoevaluación de la gestión educativa que involucra a 
diferentes actores, y con los cuales se fortalecen las instituciones educativas, entendidas como espacios sociales para la convivencia y el desarrollo de competencias en todas las áreas del conocimiento, reconociendo desde sus propios contextos sus fortalezas y debilidades para responder con un modelo pedagógico acorde a las necesidades.

\section{Aproximación a la comprensión de las percepciones}

Las percepciones tienden a tomar diversas connotaciones, sobre todo en las ciencias sociales y ciencias humanas, las cuales comúnmente suelen conocerse por concepciones, representaciones, imaginarios y entendimiento. Pero, para efecto del presente estudio, las percepciones se asumen desde tres perspectivas diferentes: la social, la educativa e individuales y colectivas. Desde la percepción social, se busca conocer y comprender a otras personas (Barón \& Byrne, 2005), pero también, busca la comprensión de los fenómenos sociales con relación entre individuos y sociedad (Vander-Zanden, 1990). Desde este punto, la percepción social se concibe como un proceso a través del cual, las personas comprenden su entorno, actúan en consecuencia a los impulsos que reciben. Estas posiciones sobre percepción social, permite estudiar a las personas y grupos frente a fenómenos complejos e interconectados con otros fenómenos sociales. En este estudio, se utilizó el concepto de percepción social como base para comprender fenómenos vinculados a la participación docente y a las prácticas docentes en el proceso de mejoramiento institucional.

En el ámbito educativo, las percepciones se adquieren mediante procesos de aprendizaje asociativo e interacción social, siendo producto de la construcción del mundo. El ser humano las utiliza para tomar decisiones, recordar, interpretar, predecir y controlar sucesos (Nespor 1987; Pajares 1992; Solar \& Díaz 2009). Por otro lado, Contreras (1998) considera las percepciones como un conjunto de posiciones que una persona o docente asume, frente a su práctica en tópicos relacionados con la enseñanza y aprendizaje de cualquier área. Aunque en estas áreas son complejas, existen medios e instrumentos que las puedan evidenciar y sintetizar como la práctica pedagógica y los elementos inmersos en ellas.

Por otra parte, es fundamental ahondar sobre las percepciones colectivas, que proporciona a los participantes la experiencia inmediata de nuevas realidades, por medio de pautas estipuladas de actuación de conductas múltiples, muy evocativas, simultáneas e integrativas. De igual forma pueden converger en procesos de percepción colectiva relaciones sociales ambiguas que implican la condensación de opuestos, basadas en mecanismos autorreferenciales que proveen un contexto inasible para la reevaluación de las relaciones que constituyen el mundo social de los participantes (Andersson \& Strander, 2004). Para efectos de esta investigación, las percepciones individuales y colectivas sobre los Planes de Mejoramiento Institucional, son relevantes en la medida en que permiten generar coherencia entre lo que el docente percibe de su participación en los procesos de transformación, lo que 
realmente necesita para su crecimiento profesional y lo que se percibe públicamente en los PMI que están en vigor.

\section{Una mirada a la autoevaluación institucional}

Según el MEN (2004) la autoevaluación institucional es un proceso autónomo y colectivo de reflexión y análisis de los objetivos institucionales, con el objeto de examinar y valorar cómo se va progresando hacia las metas fijadas por la propia institución y en qué forma se deben capitalizar los factores favorables o enfrentar los aspectos débiles, superar logros y responder con mayor efectividad a las necesidades y requerimientos de la comunidad educativa. Además, implica recoger y organizar información que permita lograr una mejor comprensión de la situación que vive la institución en un momento dado. En los lineamientos del MEN (2004), se enfatiza que "la autoevaluación al ser un proceso puede hacerse en determinados tiempos y sobre un tópico o aspecto preciso (situación financiera) o sobre la entidad en su conjunto, de una manera global e integral" (pág. 13).

El análisis de los requerimientos dados por el MEN sobre la elaboración, ejecución y evaluación del PEI, constituye los antecedentes preliminares a tener en cuenta para la elaboración del plan de mejoramiento institucional, a partir del proceso de evaluación de resultados que se realiza periódicamente y que constituye el fundamente para determinar las acciones para el mejoramiento. Entre estos planes existe una relación lógica y dialéctica que garantiza la realización eficiente de las acciones para el mejoramiento las cuales fortalecerán el PEI en el marco del proceso de transformación y perfeccionamiento educativo.

El Ministerio de Educación define la evaluación como el análisis cuantitativo y cualitativo de los resultados obtenidos al término del ciclo anual de mejoramiento continuo, cuyo propósito es obtener información relevante y objetiva con relación al logro de las metas y objetivos de la fase de desarrollo establecida. (MEN, 2008). En este sentido, la información y conclusiones que surgen del proceso anual de evaluación posibilitarán la toma de decisiones en torno a las adecuaciones y reformulaciones necesarias al Plan de Mejoramiento Institucional del año siguiente. También, se constituyen como insumo básico para la elaboración del diagnóstico institucional del siguiente ciclo de mejoramiento continuo.

\section{El Plan de Mejoramiento Institucional (PMI)}

Se concibe como la acción concreta y real que le corresponde realizar a la comunidad educativa para conseguir la educación de calidad que necesitan los estudiantes; con el fin de tener mejores aprendizaje y competencias; beneficiando a toda la comunidad educativa (MEN, 2004). Para Acosta \& Ozuna (2015); Calvo (1996); el PMI es un conjunto de metas, acciones, procedimientos y ajustes que la institución educativa precisa y pone en marcha en períodos de tiempo definidos, para que todos los aspectos de la gestión de la institución 
educativa se integren en torno a propósitos comúnmente acordados y apoyen el cumplimiento de su misión académica.

Las fases de desarrollo del mejoramiento institucional siguen tres etapas que se repetirán periódicamente, puesto que son parte del ciclo del mejoramiento continuo. Éstas contienen, a su vez, pasos y actividades, cuya realización debe conducir a resultados precisos que permitirán avanzar entre las etapas que lo definen (MEN, 2008). La primera etapa de la ruta del mejoramiento continuo es la autoevaluación institucional. Este es el momento en el que el establecimiento educativo recopila, sistematiza, analiza y valora información relativa al desarrollo de sus acciones y los resultados de sus procesos en cada una de las cuatro áreas de gestión. Con ello es posible elaborar un balance de las fortalezas y oportunidades de mejoramiento, las cuales serán la base para la formulación y ejecución del plan de mejoramiento; la segunda etapa consiste en la elaboración del plan de mejoramiento que define objetivos, actividades, tiempos y responsables de cada tarea, de manera que se logren los propósitos acordados para cada una de las áreas de gestión; y la tercera etapa consiste en el seguimiento periódico al desarrollo del plan de mejoramiento, con el propósito de establecer cuáles fueron los resultados obtenidos, las dificultades y retrasos en la ejecución, los recursos utilizados y las razones por las cuales no se realizaron ciertas actividades. Esto permitirá revisar el logro de las metas y de los objetivos, así como efectuar los ajustes pertinentes.

Por lo tanto, en este estudio se considera que el plan de mejoramiento es un proceso cíclico, dinámico, cambiante y dialéctico porque se sustenta sobre las bases del desarrollo institucional, a partir del análisis de las deficiencias detectadas en la evaluación y la necesidad de reelaborar nuevas, para lo cual también es preciso el seguimiento sistemático a las acciones planificadas en cada etapa. Los pasos de cada una de las etapas de la ruta de mejoramiento los resume Acosta \& Ozuna (2015) de la siguiente forma: Autoevaluación, elaboración del plan de mejoramiento, el seguimiento y evaluación.

\section{La Participación de docentes y directivos en los PMI}

La Ley General de Educación expedida en 1994, determina que los maestros de Colombia deben ser formados para el ejercicio de la autonomía intelectual en una cultura pedagógica, universal y nacional. Desde este enfoque, lo pedagógico y lo didáctico se articulan para que al docente se le abra un camino que dinamice su actuar en múltiples campos: el cotidiano, el experimental, el conceptual y el comunitario. Igualmente se muestra la necesidad de que el maestro sea un analista de los procesos de formación, aprendizaje, educación y enseñanza.

Para que un docente participe idóneamente requiere unos procesos de fortalecimiento e integración educativa, que le sirvan como fundamento para participar en el diseño, ejecución y transformación de los PMI. Ahora, para que los docentes y directivos fomenten de una manera adecuada la práctica de la enseñanza o cualquier otro quehacer educativo, requiere 
abrir espacios de formación de maestros, que les permita el desarrollo de una pedagogía avanzada, posibilitando una formación más justa, más equitativa y más humana; mediante el desarrollo de programas de formación, que contribuya con el desarrollo personal y profesionalmente de los maestros, generando una apertura al cambio y abiertos a las innovaciones pedagógicas" (Acosta \& Osuna, 2015).

Sobre la participación de los docentes en los PMI, Acosta y Osuna (2015) sostienen que “la renovación pedagógica, de los docentes es un aspecto necesario en el análisis de los PMI lo cual resulta trascendental para lograr los objetivos de formación que han sido planteados en los Proyectos Educativos Institucionales". Estos planteamientos apuntan hacia la necesidad de formar a docentes y directivos docentes, en la apropiación e implementación de Modelos Pedagógicos, acordes con los PEI, así como en estrategias que permitan su implementación en las prácticas de aula, lo cual debe estar entre las prioridades para que los PMI respondan efectivamente a las necesidades sentidas de la institución después de evaluar Ios PEl. Hay que entender que los procesos de mejoramiento parten del rol del docente, del quehacer pedagógico; los cuales toman importancia y validez al elaborar los planes de mejoramiento. También se destaca la profesionalidad del docente, su entrega y compromiso, trayendo consigo, un mejoramiento gradual en los procesos pedagógicos a nivel institucional. Así mismo, esta noción que se proyecta puede corresponderse con argumentos recurrentes y socialmente compartidos sobre el sentido y significado de la participación de los sujetos institucionales en los planes de mejoramiento a partir de las representaciones sociales que poseen sobre la realidad educativa en relación con sus necesidades e intereses.

Acorde con el planteamiento de Román (2004), que los sistemas educativos deben ser conscientes de los factores a mejorar y de la implementación de estrategias pertinentes en los diferentes espacios en que les corresponde actuar, exigen a los docentes la necesidad de participar en la definición del PMI de su institución, por ser este una estrategia, herramienta, idea, plan, trabajo, mecanismo que permite convertir las debilidades en fortalezas para que la institución mejore en varios aspectos y áreas de las que ellos son protagonistas activos. Por esta razón, el ejercicio de participación docente en el diseño de los PMI es muy importante porque permite que se apoderen y empoderen de las herramientas y las estrategias para fortalecer la excelencia en cada uno de los procesos, es decir, la organización con una unidad de criterios y un trabajo en equipo.

\section{Metodología}

La investigación es cualitativa, con diseño metodológico de investigación-acción (IA), proyectando un proceso cíclico de aproximación, reflexión, aplicación y evaluación (Kemmis \& McTaggart, 1988). La población participante la constituyen 39 docentes y 4 directivos 
docentes, con representatividad de preescolar, básica primaria, secundaria y media académica. Se utilizaron como instrumentos actas de reuniones, guion o cuestionario de preguntas, rejilla de anotaciones y la construcción de formatos oficiales sobre los PMI aceptados y aplicado por los docentes.

En cuanto al diseño de la Investigación se tuvieron en cuenta las fases de la investigación propuestas por Kemmis y McTaggart (1988), donde inicialmente se caracteriza el problema en forma conjunta (aproximación), luego se plantea el plan de acción (reflexión), posteriormente se ejecutan las acciones acordadas (aplicación) y finalmente se valoran los resultados (evaluación):

Fase de aproximación. En esta fase se hace una revisión y análisis de los Planes de Mejoramiento Institucional y su funcionalidad; luego se identifican las percepciones que tienen los docentes sobre ellos, a partir de sus voces, y la aplicación de la entrevista semiestructurada. En este contexto surgen la descripción del problema y los propósitos iniciales de la investigación.

Fase de reflexión. En esta fase se diseñó el plan de acción, resultado de la discusión conjunta en los grupos de discusión. En este espacio se ideó la organización de los grupos y la asignación de funciones autónomas, para la elaboración del trabajo. Se realizó el cronograma de acción, y su dinámica permitió el fortalecimiento del marco teórico.

Fase de aplicación. Se implementa el plan de acción, relacionado con el acercamiento a la realidad de los planes de mejoramiento Institucional y su relevancia en las instituciones educativas; se realizan los talleres sobre nuevos enfoques a los PMI.

Fase de evaluación. Esta fase permite la compilación de las acciones y resultados obtenidos a lo largo de las fases de estudio; resaltando las lecciones aprendidas hasta el momento, se identificaron los aspectos por mejorar y se emitieron los resultados alcanzados y los emergentes, que permiten la adopción de una postura diferente, frente al desarrollo de los planes de mejoramiento institucional.

\section{Resultados}

Los resultados de la investigación se muestran de acuerdo con cada una de las fases propuestas en el diseño metodológico:

\section{Fase de aproximación}

En esta fase se hizo un análisis de los resultados que giran alrededor de la revisión documental de los PMI, el grupo de discusión y la entrevista semiestructurada, que dan cuenta 
sobre las percepciones y nivel de participación de los directivos y docentes sobre los Planes de Mejoramiento Institucional.

Las percepciones de los docentes y directivos docentes respecto a los Planes de mejoramiento Institucional (PMI), generan respuestas variadas; un primer grupo conformado por 16 participantes, tiende a manifestar que el PMI es una herramienta útil, que les permite reflexionar sobre las debilidades encontradas a la autoevaluación institucional; un segundo grupo de 11 docentes, aunque lo perciben como instrumento benéfico, deja entrever dudas con relación a su operatividad, "siempre y cuando se lleve de la manera que debe ser" (DEV3). Y un tercer grupo con la participación de 16 docentes, lo ve como un simple documento para cumplir requisitos. De esta manera, la diversidad de percepciones tanto teórica como conceptual sobre los PMI, tienen implicaciones positivas y negativas; la primera tiene una mirada plural para la construcción en el consenso y desde diferentes puntos de vista, la mejora de la problemática que se presenta; en cambio la segunda, muchas veces se mantiene inamovible que no da apertura a la generación de cambios en la actitud del docente y se detengan los procesos.

El análisis de esta información condujo a la generación de propuestas que conllevaran al fortalecimiento; primero de realizar la evaluación institucional y a partir de allí realizar los Planes de Mejoramiento Institucional. Pero se ideó conformar un grupo de discusión desde la Investigación -Acción, considerando que estos, son distinguidos como una técnica específica de la investigación cualitativa, orientada a la obtención de información, dentro de la categoría más amplia de entrevistas grupales.

Mediante el grupo de discusión se pudo identificar algunas percepciones que tienen los docentes sobre los Planes de Mejoramiento Institucional a través de las inquietudes y necesidades que suscitaron en las intervenciones de cada participante; en primer lugar, lo perciben como una herramienta útil para la mejora institucional, dejando entrever una postura clara acerca de los PMI, manifestando “...quien practica el mejoramiento progresa"....de ahí que los PMI son una herramienta y una acción para conseguir una meta...cuando planea se organiza la ruta que debemos mejorar" (DDP7). Aquí se infiere que los PMI se aprecian como una competencia institucional para la mejora continua, “....por eso el PMI de la institución sirve como herramienta... si se ejecuta y evalúa correctamente.... otros colegios, si le ha puesto atención y han mejorado..." (DEV9) esto coincide con los fundamentos de MEN, (2004) le da un valor importante a los PMI como herramienta que posibilita y orienta el rumbo de la Institución Educativa.

Por otra parte, se ratifica la mirada de los docentes sobre los PMI, quienes lo ven como un instrumento de reflexión, el cual abre campo al análisis profundo en la forma de actuar frente a las dificultades escolares, ante ello, un docente expresa: “... permite ... mirar como estoy llevando a cabo mi proceso de enseñanza..., pensar si estoy encaminando...esto hace que autorreflexione" (DEV11). Aquí se vislumbra en el participante un estado "doliente" frente 
al proceso formativo y se dispone a participar en la solución de las inquietudes generadas alrededor del grupo de discusión

Otro tipo de percepción docente mirado en el grupo de discusión se enfoca al ámbito teórico; "Desde mi óptica los PMI han sido buenos teóricamente, pero si se aplica como debe ser...pero, en la práctica no se muestran resultados..." (DEV11). Cada argumento desde la visión de los docentes, muestra una realidad panorámica de las situaciones institucionales, si bien existen docentes considerados de alta calidad, que muestran un gran fundamento y tienen la voluntad de hacer el ejercicio en el desarrollo de los Planes de mejoramiento; las condiciones y la escases de algunos recursos importantes, hacen que se reflejen situaciones poco adecuadas en el recinto institucional, como alto índice de deserción, la aparición de la drogadicción, la violencia escolar, la aparición de niños embarazadas y sobre todo el matoneo como la agresión entre iguales. Pero, si a los planes de mejoramiento se les nutre con los recursos necesarios en cierta medida cambiará la situación adversa.

Finalmente, algunos docentes perciben los PMI desde la incertidumbre cultural; es decir, se vislumbra un panorama diferente y percibe los planes de mejoramiento institucional como un requisito que debe cumplir a los entes territoriales como requisito al cumplimiento de unas acciones; por eso, docentes del grupo focal argumentan que: "Realmente el plan de mejoramiento no pasa del papel..." (DEP7), la mirada cultural del docente, muestra la elaboración de los PMI como "una perdedera de tiempo y viven echándole la culpa al Estado..." (DEP8).

En síntesis, se demuestra que algunos docentes de los establecimientos educativos requieren tener claridad tanto conceptual como operativa, para ir minimizando las percepciones que tienen sobre los Planes de Mejoramiento Institucional; y pueda convertirse en un actor que coadyuve al fortalecimiento de los procesos y gestiones de los entornos escolares.

\section{Fase de reflexión (Plan de acción)}

Esta fase dio respuesta al propósito relacionado con el diseño del plan de acción, de la metodología I-A, que emerge a partir de los insumos recolectados en el grupo de discusión de docentes y directivos docentes. El plan de acción se concretó en forma conjunta a partir de la realización de las siguientes acciones a seguir:

a) Taller sobre documentación de la guía 34, mediante la dinámica de grupos "dolientes", quienes se encargan de ilustrar en los componentes que les corresponde a los docentes seleccionados para el trabajo en una gestión. El líder de grupo orienta los fundamentos de la guía 34 y ejecuta las acciones de la gestión asignada. 
b) Apropiación de los fundamentos de los PMI, para el empoderamiento de la gestión y la adquisición de la formación sobre los Planes de Mejoramiento Institucional, desde la perspectiva educativa.

c) Manejo significativo de los instrumentos, para plasmar la realidad en la gestión asignada para cada grupo, de ahí que se hace necesario entenderlo y dinamizarlo.

d) Seguimiento periódico al PMI, mediante una revisión conjunta, que permita el monitoreo y garantice la ejecución de las acciones plasmadas para el mejoramiento.

\section{Fase de aplicación}

Una vez definido el plan de acción se acordó empezar por la organización de los grupos que lideraron las diferentes gestiones y la apropiación de los talleres realizados. Dentro de la dinámica de trabajo, se realizaron ejercicios compartidos sobre la comprensión ilustrada de los diferentes componentes de las gestiones a las cuales se les elaborarían los Planes de Mejoramiento institucional. Algunos docentes mostraron sus posturas sobre el particular e identificaron algunos puntos débiles relacionados con su percepción de los PMI y la autoevaluación institucional.

En este mismo espacio, se retoma la formación, liderada por cada coordinador y responsables de grupo, a la apropiación de los fundamentos de los PMl; de tal manera que los docentes, le den sentido a la elaboración de los PMI; se apropien de sus dinámicas teóricas y operativas para hacer más agradable el trabajo. Para lo cual, el líder en forma conjunta con los docentes de apoyo, participan en una charla sobre este tema. Cada líder asignado a una gestión tuvo la autonomía y responsabilidad que se ejecuten las acciones pactadas en el PMI. Este trabajo, permitió el empoderamiento de la gestión asignada, pero al mismo tiempo adquirieron la formación sobre los Planes de Mejoramiento Institucional.

Durante la socialización del taller para la apropiación de los fundamentos del PMI, se destaca como aspectos puntuales, la dinámica para el ejercicio de los planes de mejoramiento institucional; teniendo en cuenta ¿Cuáles son sus instrumentos? ¿Cuál es su ruta de implementación? ¿Por qué es importante un seguimiento periódico al PMI? Estos interrogantes permitieron al líder de cada grupo de gestión dinamizar una socialización a manera de charla, para clarificar los aspectos abordados.

La puesta en marcha de procesos de mejoramiento escolar requiere que el equipo de gestión conformado por diversos integrantes de la comunidad educativa y liderada por el rector o director se organice para realizar cada una de las actividades previstas. Esta tarea tiene que ver tanto con la conformación de grupos de trabajo y la definición de responsables, como con el ordenamiento de las tareas y el establecimiento de tiempos y plazos para realizarlas. Para apoyar este trabajo en los establecimientos educativos, de manera que sea eficiente y productivo, se propone una ruta de tres etapas que se repetirán periódicamente, 
puesto que son parte del ciclo del mejoramiento continuo. Éstas contienen, a su vez, pasos y actividades, cuya realización debe conducir a resultados precisos que permitirán avanzar a la etapa siguiente.

La reflexión en torno a este aspecto permitió abrir espacios claros para la implementación y elaboración de los PMI, tanto en la institución educativa Policarpa Salavarrieta como la institución educativa Victoria Manzur; al finalizar el taller se concluyó que el PMI, es importante porque apuntan a los intereses de los establecimientos educativos y es el indicador que emite el concepto si se está o no mejorando. La mayoría de los docentes manifestaron haber comprendido los fundamentos del PMI y que desde el momento empezarían a darle un nuevo sentido a lo que plasmarían en él. Igualmente se empezó a evidenciar el adecuado manejo de los instrumentos como soporte para plasmar en ellos la realidad en la gestión asignada para cada grupo y elaborar su ruta de seguimiento como espacio para reflexionar en forma conjunta sobre lo logrado y ejecutar los procesos de realimentación al proceso.

Con la fundamentación realizada a los docentes se procedió a la realización de Planes de Mejoramientos Institucionales significativos, en la que fue evidente la alta participación de los docentes; mostrando en cierta manera "rivalidades cognitivas y procesuales" en la elaboración y dinámicas dialógicas en la construcción de los PMI. A partir del diálogo y la reflexión entre docentes y directivos sobre la manera como anualmente se realiza la autoevaluación institucional y el insignificante sentido de formular planes de mejoramiento que poco o nada generan transformaciones en la realidad educativa de la institución, se llegó a la conclusión de que se hacía necesario resignificar el PEl, mediante la dinámica de la autoevaluación institucional y los PMI.

El desarrollo de los planes de mejoramiento, mediante esta dinámica, tuvo su efecto positivo, debido a que durante su elaboración se observó el aporte masivo y grandes discusiones frente a la forma de abordar las acciones para el mejoramiento de los procesos de cada gestión; finalmente, hacia al inicio de 2019 se realizó un nuevo análisis valorativo a los PMI, los cuales se muestra a continuación:

La indagación y reflexión sobre los PMI y sobre todo la participación del docente, permitió observar la gran necesidad que tienen los establecimientos educativos focalizados, de darle un nuevo sentido a las prácticas rutinarias dadas a los PMI. Esto genera como inquietud, que las comunidades educativas deben realizar encuentros para reflexionar acerca de los diferentes problemas que tienen los establecimientos educativos en cuanto a su vida académica, directiva, comunitaria y administrativa y financiera. En este espacio, fue evidente la necesidad de una fundamentación teórica de los procesos contemplados en la guía 34, con relación a la autoevaluación institucional, las gestiones institucionales, el levantamiento de los planes de mejoramientos, su dinámica operativa, instrumentos usados y la necesidad de propender por un seguimiento efectivo, que conduzca a la mejorar. 
La reflexión sobre la guía 34, justificó la necesidad de replantear no solo la forma de elaborar los PMI, sino ir más allá, es decir a la revisión del PEl y su actualización, el cual contiene los componentes esenciales que guían la vida de una institución educativa. Se recalca en el trabajo investigativo la necesidad de introducir cambios en el diseño de los Planes de mejoramiento Institucional, teniendo en cuenta las necesidades pedagógicas, didácticas y normativas que lo deben regir de acuerdo con los resultados de la valoración, donde se evidenciaron oportunidades de mejora en los PMI.

Los docentes expresaron con especial interés y preocupación el hecho que no se esté contemplando en el PEI, la dinámica operativa de las gestiones que valoran la evaluación institucional como el poco interés por la inclusión y el apoyo pedagógico a estudiantes en situación de vulnerabilidad que experimentan barreras al aprendizaje y a la participación, puesto que en la institución hay varios estudiantes con esta condición, que están siendo atendidos y evaluados con criterios no muy claros que no se ajustan a las necesidades individuales de cada caso, por esta razón se hace necesario que la institución acuerde mecanismos de atención pedagógica evaluativa para brindar un proceso incluyente que genere espacios de participación e interacción de todos los estudiantes en condiciones de equidad, pero que deben ser direccionados desde el PMI.

\section{Resignificación de los planes de mejoramiento institucional}

El grupo investigador, se reunió con el grupo de discusión y acordaron la generación de acuerdos de autoformación por gestiones, para posteriormente hacer ajustes a los PMI. Para ello, hicieron análisis a las acciones programadas en los PMI entre 2017 y 2018; seguido a ello, se socializaron los hallazgos, que fueron expuestos en un encuentro de docentes (grupo de discusión). En este espacio, emergió la iniciativa de organizar las actividades por etapas, a fin de que los Planes de mejoramiento Institucional que se realizarían, tuvieran una forma diferente de diseñarlo y adoptarlo.

La realización de este proceso generó una dinámica diferente para su adopción; conllevando al grupo de cada gestión dinamizar procesos de autoformación por gestiones, para posteriormente hacer ajustes a los PMI y socializarlos en los encuentros de docentes. A manera de síntesis permitió:

- La realización de ejercicios compartidos sobre la comprensión ilustrada de los diferentes componentes de las gestiones de los Planes de Mejoramiento institucional

- Demostración docente de una postura particular, que les conlleva a la identificación de algunos puntos débiles relacionados con su percepción de los PMI y la autoevaluación institucional. 
- La generación de estrategias pedagógicas que activan la reflexión colectiva e individual, alrededor de las gestiones directiva, académica, comunitaria y administrativa y financiera, para abordar los PMI de los establecimientos educativos.

- Apropiación teórica y operativa para hacer más significativo la elaboración de los PMI y su proceso de seguimiento. Para ello se establece una ruta en tres etapas que se repetirán periódicamente, puesto que son parte del ciclo del mejoramiento continuo. Éstas contienen, a su vez, pasos y actividades, cuya realización debe conducir a resultados precisos que permitirán avanzar a la etapa siguiente.

La reflexión en torno a este aspecto permitió abrir espacios claros para la implementación y elaboración de los PMI, tanto en la institución educativa Policarpa Salavarrieta como la institución educativa Victoria Manzur; al finalizar el taller se concluyó que los PMI, son de gran importancia porque apuntan a los intereses de los establecimientos educativos y es el indicador que emite el concepto si se está o no mejorando. La mayoría de los docentes coinciden haber comprendido los fundamentos del PMI y que desde el momento empezarían a darle un nuevo sentido a lo que plasmarían en él.

\section{Fase de Evaluación}

Esta fase valora los avances generados en las acciones implementadas y el cumplimiento de los objetivos planteados, para ello se toma la información derivada de los instrumentos utilizados. Con relación a las percepciones que tienen los docentes sobre los Planes de Mejoramiento Institucional, surgen las siguientes apreciaciones:

Los docentes conciben los PMI como una herramienta útil para la mejora institucional, porque son instrumentos direccionados al alcance de los propósitos visionados, "...... Ios PMI son una herramienta y una acción para conseguir una meta...cuando planea se organiza la ruta que debemos mejorar" (DE9). En este sentido el MEN, (2004) ratifica que es una herramienta con la cual es posible orientar el rumbo de la Institución Educativa, a partir de su caracterización hacía unos propósitos y resultados previamente acordados, y liderado por el equipo de gestión institucional. Además, se expresa que "incluye un conjunto de metas, acciones, procedimientos y ajustes que la I.E. define y ejecuta todos los aspectos que en ella se integren, en torno a propósitos comúnmente acordados y conduzcan al cumplimiento de su misión" (MEN, 2004, pág. 8). Para Foucault (1975), se convierte en una poderosa herramienta, útil no solo para explicar y analizar los diferentes aspectos de la realidad sino también para redescubrirlo como articulador de las nociones de poder y saber.

Otro grupo concibe los PMI como instrumento de reflexión, que abre campo a la manera de actuar frente a las dificultades escolares, "EI PMI, me permite pensar, como hacer mejora las cosas y determinar cuáles no me han funcionado y cuales sí; desde esta perspectiva, uno reflexiona y adapta la enseñanza con mejores opciones" (DE11), desde esta mirada, permite 
al docente cambiar en la marcha procesos que, si bien son buenos, pero no son pertinentes; además, despierta la sensibilidad sobre el problema que emerge a la institución o al estudiante. De ahí que el PMI, según los docentes es un instrumento que permite dialogar con la localidad, el ente territorial, otras instituciones y ella misma al interior de su comunidad. Por tanto, es necesario hacer reflexión sobre la acción, de lo contrario no será posible articular un debate comprensivo y transformador.

Los PMI vistos desde el ámbito teórico, se concibe como una herramienta compleja, que el Ministerio de Educación Nacional le propone para que organicen lo relacionado con las gestiones que demanda una institución educativa, que reflexione a través de su accionar; pero el mismo docente se enfrenta con dificultades que le impiden trabajar cómodamente. Se infiere que la relación gestión escolar con gestión participativa, incluye y compromete a la comunidad educativa en un conjunto de acciones que constituirían el PMI con una intencionalidad clara como lo es la mejora institucional, con base en unos indicadores definidos y concertados (Ojeda \& Romero, 2014)

Los PMI vistos desde la incertidumbre cultural. El diseño, la ejecución, la evaluación y la realimentación de los PMI, derivan tensiones y dificultades al momento de su ejecución en los establecimientos educativos; desde esta mirada, se destacan aspectos relacionados con la gestión de tiempo, el interés de los entes gubernamentales; el afán de los directivos de mostrar el trabajo y los intereses individuales de los docentes y las instituciones educativas desde el punto de vista administrativo (Quiroga, 2014). Acá el docente, vislumbra un panorama diferente y percibe los planes de mejoramiento institucional como un requisito que debe cumplir a los entes territoriales como requisito al cumplimiento de unas acciones; por eso, docentes del grupo focal argumentan que:

"Realmente los planes de mejoramiento no pasa del papel, ojala pasen a la acción; por qué digo esto, simplemente porque el PMI se hace al final del año muchas veces como docente bueno los que queremos hacerlo rápido para poder irnos y muchas veces por esa ligereza que no se hace bien hecho... otro aspecto, siempre estamos hablando del directivo, como responsable de eso... ahora considero que el plan de mejoramiento depende de cómo sea direccionado desde la parte directiva y a toda la organización" (DE15).

Desde este punto de vista, es notable una incertidumbre del docente frente al ejercicio de los Planes de Mejoramiento Institucional, lo cual da validez al fundamento teórico en su diseño, pero operativamente limitado. Esto hace que el docente culturalmente adopte una cultura longitudinal de año tras año, repartiendo los mismos procesos sin evidenciar sus resultados. De ahí, que al momento de realizar los PMI se muestren desinteresados por su desarrollo, considerándolos una perdedera de tiempo y echándole la culpa al Estado.

Con relación a los procesos académicos, fundamentalmente en las prácticas pedagógicas, expresan que el PMI los ha llevado a reconsiderar su ejercicio pedagógico y replantar el proceso en el aula; como también a tomar nuevas posturas cambiantes frente a las situaciones que presenta debilidad. Otro gran aporte, surge en la entrevista, en la que los 
docentes expresan que los PMI han impactado en los establecimientos educativos, porque se evidencias trasformaciones en su interior; mientras que otros, lo ven gradualmente, argumentando que han funcionado parcialmente.

Respecto a la participación de los docentes en el ámbito educativo y la elaboración de los Planes de mejoramiento institucional, estos dan cuenta de su alta participación en la elaboración, ejecución y evaluación de los PMI; pero que existen algunos, que se limitan al trabajar y otros que realmente no aportan nada al mejoramiento.

A nivel general, la información derivada de la entrevista y el grupo de focal determinó la percepción docente sobre los PMI, conllevando al grupo investigador a la generación de una propuesta direccionada al fortalecimiento, a través de la autoevaluación institucional, y partir de los resultados realizar Planes de Mejoramiento Institucional dinámicos, lo cual permitió el surgimiento de un grupo focal, para su análisis, interacción y discusión hacia la búsqueda de las posibilidades de exploración y de generación espontánea de información.

\section{Conclusiones}

Los resultados de la investigación de la cual se deriva este artículo permiten que considerar que la Investigación-Acción (IA) permite la integración espontánea de los directivos y docentes, así como propicia espacios para la reflexión y la generación de conocimiento a partir de sus voces; expresando sus debilidades y fortalezas, lo cual enriquece los saberes con la participación de todos, frente a un estudio que requiere cambios en la manera de concebir los planes de mejoramiento institucional.

Las informaciones develadas mediante la entrevista y el grupo focal permitieron evidenciar los puntos críticos de la dinámica para la elaboración, ejecución y evaluación de los PMI, contrastadas con las voces que proporcionaron los participantes en aras de formular acciones de mejoramiento institucional. La diversidad de percepciones tanto teórica como conceptual sobre los PMI, tienen implicaciones positivas y negativa: la primera tiene una mirada de la diversidad de posturas para la construcción en el consenso y desde diferentes puntos de vista, la mejora de la problemática que se presenta; en cambio la segunda, muchas veces se mantiene inamovible que no da apertura a la generación de cambios en la actitud del docente y se detengan los procesos

La implementación de la entrevista a docentes y directivos, y la dinámica del grupo de discusión, permitieron dar cuenta del propósito relacionado con la identificación de las percepciones y nivel de participación que tienen los directivos y docentes sobre los Planes de Mejoramientos Institucionales, la cual es vista como una herramienta útil para la mejora institucional, destacando su importancia como instrumento direccionado al alcance de los propósitos visionados. Aquí se percibe como una competencia institucional, para el desarrollo 
de las competencias a la mejora de la educación que se imparte en cada establecimiento educativo, tal como lo indica el MEN, (2004) considerándola una herramienta con la cual es posible orientar el rumbo de la Institución Educativa, a partir de su caracterización, propósitos y resultados previamente acordados, y liderado por el equipo de gestión institucional. También los PMI son percibidos como un instrumento de reflexión, asumida desde ópticas diferentes, la cual abre campo a la reflexión sobre la manera de actuar frente a las dificultades escolares. Esta reflexión, permite repensar las cosas, y determinar las posibles rutas de solución y adaptar los procesos hacia esas reflexiones.

Continuando con este enfoque, algunos docentes, perciben el PMI, como un referente teórico, que dista de los procesos prácticos, es decir, teóricamente están bien fundamentados, pero al momento de llevarlos a la práctica, sucumben en su intento, mostrándose limitados por diversos factores operativos. Finalmente, son visto como una incertidumbre cultural, cuyo diseño, ejecución, evaluación y la realimentación, originan tensiones y dificultades al momento de su ejecución, porque desde la mirada docente, se vuelve monótono y repetitivo, vislumbra un panorama diferente, percibiéndolos como un requisito que deben cumplir a los entes territoriales como requisito al cumplimiento de unas acciones. Esto hace que el docente culturalmente adopte una cultura longitudinal de año tras año, repartiendo los mismos procesos sin evidenciar sus resultados.

Por otra parte, la operatividad de los PMI y su relación con las prácticas pedagógicas; se muestran con un matiz de mejora en su dinámica, por la reflexión y la ejercitación pedagógica, que permite replantar el proceso en el aula; como también a tomar nuevas posturas cambiantes frente a las situaciones que presenta debilidad, lo cual muestra gradualmente algunas transformaciones al interior de la institución.

Con relación a la participación de los docentes en el ámbito educativo y la elaboración de los Planes de mejoramiento institucional, estos dan cuenta de su alta participación en la elaboración, ejecución y evaluación de los PMI, pero que existen docentes, que se limitan al trabajar y otros que realmente no aportan nada al mejoramiento. En cuanto a la caracterización de los planes de mejoramiento, se evidencia que en las diversas gestiones existen pocos aciertos y muchos desaciertos, porque el plan de acción derivado de los PMI, pocos son los que se llevan a cabo, debido al poco seguimiento a las acciones de apoyo y nivelación a las gestiones evaluadas.

Con referencia al diseño y la implementación del plan de acción, para el mejoramiento de los PMI, una vez analizados los datos, se evidenció la necesidad de diseñar un plan de acción relacionado con la fundamentación del PMI, los instrumentos y el desarrollo del plan de seguimiento. En este sentido, su desarrollo permitió la creación de una concepción diferente sobre el trabajo de los planes de mejoramiento, dándole sentido, dinamismo y una nueva forma para proceder en su realización. Por tanto, es posible concluir que la resignificación de los planes de mejoramiento institucional, generó una dinámica diferente para su adopción, conllevando al grupo de cada gestión dinamizar procesos de autoformación 
por gestiones, para posteriormente hacer ajustes a los PMI y socializarlos en los encuentros de docentes a través del grupo focal.

\section{Referencias}

Acosta, M., \& Osuna, J. (2015). Significación del plan de mejoramiento institucional, en la institución educativa rural Luis Antonio Duque Peña, de la ciudad de Girardot -Cundinamarca. Girardot -Cundinamarca: Gestion Educativa.

Andersson, B., \& Strander , K. (2004). Percepciones de la escuela y futura adaptación a la vida: un estudio longitudinal entre las edades de 18 y 25 años. Revista escandinava de investigación educativa, 459-476.

Altablero. (09 de 2007). La gestión educativa es la vía al mejoramiento de la educación.: https://www.mineducacion.gov.co/1621/article-137440.html

Avaca, P., \& De León, E. (2012). Planes de mejora institucionales: ¿se constituyen en verdaderos desafíos para las escuelas secundarias? un abordaje evaluativo. San Luis Argentina: Universidad Nacional de San Luis.

Barón, R., \& Byrne, D. (2005). Psicología social. México: Pearson. Prentice Hall.

Calvo, G. (1996). Los proyectos educativos institucionales . Recuperado el 22 de 05 de 2019, de revistas.pedagogica.edu.co:

http://revistas.pedagogica.edu.co/index.php/RCE/article/download/5400/4427

Castañeda, E. (2009). Las condiciones sociales para el aprendizaje en la relación equidad social y educación. En N. López, De relaciones, actores y territorios: hacia nuevas políticas para la educación en América Latina. Buenos Aires (Argentina): IIPE-UNESCO Sede Regional Buenos Aires.

Castañeda, A. (2015). Planes de mejoramiento institucional, pensados desde el liderazgo que implica la gestión educativa. Manizales - Caldas: Universidad de Manizales.

Fundación Empresarios por la Educación . (2018). Ideas para tejer: Reflexiones sobre la educación en Colombia 2010-2018. Bogota Colombia: Fundación ONG.

Kemmis, S., \& McTaggar, R. (1988). Cómo planificar la investigación acción. Barcelona: Laertes.

Nespor, J. (1987). El papel de las creencias en la práctica de la enseñanza. Revista de estudios curriculares, 317-328.

Pajares, M. (1992). Creencias de los docentes e investigación educativa: limpiar una construcción desordenada. Revisión de la investigación educativa, 307-332.

MEN. (1994). Ley General de la Educación.

MEN. (2004). Planes de Mejoramiento ¿Y ahora Cómo mejoramos? Guia $n^{\circ} 5$. Planes de Mejoramiento ¿Y ahora Cómo mejoramos? Bogotá, Colombia: Impresiones Periódicas S.A. 
MEN. (2008). Guía para el mejoramiento institucional de la autoevaluación al plan de mejoramiento: https://www.mineducacion.gov.co/1759/articles-177745_archivo_pdf.pdf

Ojeda, K., \& Romero, S. (2014). Plan de mejoramiento Institucional como dispositivo de gestión participativa para el fortalecimiento de la calidad educativa. Cartagena: Universidad de la Costa CUC.

Quiroga, M. (2014). Liderar la implementación del plan de mejora escolar oportunidades, desafíos y dificultades. Un estudio de caso. Sevilla ( España ): Universidad de Sevilla ( España ).

Román, M., \& Murillo, F. (2004). Retos en la evaluación de la calidad de la educación en américa latina. Revista iberoamericana de educación, 97-120.

Rosero, A. (29 de junio de 2012). Percepciones y prácticas de gestión de directivos y docentes en instituciones educativas del municipio de pasto. Revista UNIMAR(59), 41-54.

Solar, M., \& Díaz, C. (2009). El profesor universitario: construcción de su saber pedagógico e identidad profesional a partir de sus cogniciones y creencias. Revista Calidad en la Educación, 207-232.

Vander-Zanden, J. (1990). Manual de psicología social. Madrid: Paidos Iberica. 